\title{
Solution Synthesis of Germanium Nanowires Using a Ge+2 Alkoxide Precursor
}

\author{
Henry Gerung ${ }^{a}$, Timothy J. Boyle ${ }^{b^{*}}$, Louis J. Tribby ${ }^{b}$, Scott D. Bunge ${ }^{b, \dagger}$, C. Jeffrey \\ Brinker ${ }^{a, b}$, and Sang M. Han ${ }^{{ }^{\star}}$ \\ aDepartment of Chemical and Nuclear Engineering, University of New Mexico, 209 Farris Engineering \\ Center, Albuquerque, NM 87131 \\ bAdvanced Materials Laboratory, Sandia National Laboratories, 1001 University Blvd SE, Albuquerque, NM \\ 87106
}

\section{Abstract}

A simple solution synthesis of germanium $\left(\mathrm{Ge}^{0}\right)$ nanowires under mild conditions $\left(<400{ }^{\circ} \mathrm{C}\right.$ and 1 atm) was demonstrated using germanium 2,6 dibutylphenoxide $\mathrm{Ge}(\mathrm{DBP})_{2}(\mathbf{1})$ as the precursor where $\mathrm{DBP}=\mathrm{OC}_{6} \mathrm{H}_{3}\left(\mathrm{C}\left(\mathrm{CH}_{3}\right)_{3}\right)_{2}-2$,6. Compound $\mathbf{1}$, synthesized from $\mathrm{Ge}\left(\mathrm{NR}_{2}\right)_{2}$ where $\mathrm{R}=\mathrm{SiMe}_{3}$ and two equivalents of DBP-H, was characterized as a mononuclear species by single crystal X-ray diffraction. Dissolution of $\mathbf{1}$ in oleylamine, followed by rapid injection into a 1-octadecene solution heated to $300{ }^{\circ} \mathrm{C}$ under an atmosphere of Ar, led to the formation of $\mathrm{Ge}^{0}$ nanowires. The $\mathrm{Ge}^{0}$ nanowires were characterized by transmission electron microscopy (TEM), X-ray diffraction analysis, and Fourier transform infrared spectroscopy. These characterizations revealed that the nanowires are single crystalline in the cubic phase and coated with oleylamine surfactant. We also observed that the nanowire length $(0.1$ to $10 \mu \mathrm{m}$ ) increases with increasing temperature (285 to 315 ${ }^{\circ} \mathrm{C}$ ) and time (5 to $60 \mathrm{~min}$ ). Two growth mechanisms are proposed based on the TEM images intermittently taken during the growth process as a function of time: (1) self-seeding mechanism where one of two overlapping nanowires serves as a seed, while the other continues to grow as a wire and (2) self-assembly mechanism where an aggregate of small rods ( $<50 \mathrm{~nm}$ in diameter) recrystallize on the tip of a longer wire, extending its length.

\section{Keywords}

Germanium; nanowire; solution synthesis

\section{BRIEFS}

Solution synthesis of germanium nanowires.

\section{Introduction}

One-dimensional (1-D) semiconductor nanowires have been actively investigated in an effort to utilize them for advanced applications such as liquid crystal displays, ${ }^{1}$ field effect transistors, 2 optical waveguides, laser diodes, and biological sensors. ${ }^{3}$ The physical properties of nanowires at their characteristic length scale, in contrast to their bulk behavior, are strongly governed by the localized electrons confined within the nanowires. 4,5 This quantum

\footnotetext{
*Author to whom correspondences should be sent: Timothy J. Boyle [Ph: (505) 272-7625, Fax: (505-272-7663, E-mail: țiboyle@sandia.gov] and Sang M. Han [Ph: (505)277-3118; Fax: (505)277-5431; Email: meister@unm.edu].

${ }^{\dagger}$ Current address: Kent State University, Department of Chemistry, 305 William Hall, Kent, $\mathrm{OH} 44242$.
} 
confinement effect enables the use of nanowires in diverse areas of electronics, optoelectronics, and biological sensing. Among group 14 elements, both carbon $(\mathrm{C})$ and silicon $\left(\mathrm{Si}^{0}\right)$ have been predominantly used for the development of advanced nanoelectronic devices. For instance, $\mathrm{Si}^{0}$ nanowires can serve simply as interconnects or as building blocks for advanced logic devices. ${ }^{2,} 3$ Preliminary results have also been reported for the use of $\mathrm{Si}^{0}$ nanowires as sensors to detect biological and chemical reagents. ${ }^{2,3}$ In comparison to $\mathrm{C}$ and $\mathrm{Si}^{0}$, the synthesis and utilization of $\mathrm{Ge}^{0}$ nanowires have been relatively scarce despite their unique characteristics.

6-10 Nanoscale $\mathrm{Ge}^{0}$ semiconductor systems are superior to $\mathrm{Si}^{0}$ for (i) higher electron and hole mobility, ${ }^{11}$ (ii) a larger Bohr exciton radius that yields a more pronounced quantum confinement effect, ${ }^{12,13-15}$ and (iii) a predicted nanostructure with direct-bandgap behavior, although bulk $\mathrm{Ge}^{0}$ is an indirect bandgap material. ${ }^{16}$

One reason for the scarce exploration of $\mathrm{Ge}^{0}$ nanowires as an advanced material is due to the lack of a simple and benign synthetic pathway to this morphology. 17 The previously reported routes cover a myriad of processes, including chemical vapor deposition (CVD) $, 6,18$ laser ablation, ${ }^{19}$ vapor-liquid-solid (VLS) growth, ${ }^{20}$ templated supercritical fluid, ${ }^{21-24}$ and combination of these techniques. The VLS technique is most widely used amongst these methods. Modified VLS techniques, such as solution-liquid-solid (SLS) and supercriticalfluid-liquid-solution (SFLS) $6,25,26,27$ growth techniques, have also been developed. While these routes can yield $\mathrm{Ge}^{0}$ nanowires, they require the use of metal catalysts (e.g., $\mathrm{Au}^{0}$ or $\mathrm{Fe}^{0}$ ) or non-metallic, high-boiling-point catalysts (e.g., trioctylamine) as seed materials to initiate the growth and to define the growth direction of $\mathrm{Ge}^{0}$ nanowires. ${ }^{28,} 29$ Also, the use of high temperature and pressure coupled with the volatility of the precursor could be potentially hazardous. ${ }^{21}$ For the synthetic routes described above, the typical Ge precursors are in the +4 oxidation state, such as $\mathrm{GeH}_{4}, \mathrm{GeI}_{4}, 7 \mathrm{Ge}\left(\mathrm{CH}_{3}\right)_{4}, 6$ or $\mathrm{GexFe}_{1-\mathrm{x}}$ alloy ${ }^{19}$. More recently, a $\mathrm{Ge}$ precursor in the ${ }^{+2}$ oxidation state has found utility in a CVD and SLS systems, using $\mathrm{Fe}^{0}$ or $\mathrm{Bi}^{0}$ as the catalyst material. ${ }^{18,30}$ Despite the success of producing $\mathrm{Ge}^{0}$ nanowires by these techniques, the presence of foreign seed particles adds complexity to the production of pure $\mathrm{Ge}^{0}$ nanowires and influences their electrical and optical properties.

In comparison, select solution routes can be simple and effective for the production of highquality $\mathrm{Ge}^{0}$ nanoparticles. To date, very limited information has been available for the production of $\mathrm{Ge}^{0}$ nanowires from these solution routes. 31,32 One reported solution route to $\mathrm{Ge}^{0}$ nanowires involves the reduction of $\mathrm{GeCl}_{4}$ and phenyl- $\mathrm{GeCl}_{3}$ by $\mathrm{Na}^{0}$ in an alkane solvent at elevated temperature and pressure. ${ }^{8}$ In this solution route, the complexity of the reaction as well as the potential contamination by metal reducing agents and salt byproducts limit the direct use of the as-produced $\mathrm{Ge}^{0}$ nanowires for novel devices. An ideal case for the synthesis of $\mathrm{Ge}^{0}$ nanowires would be a simple synthesis conducted under relatively benign conditions associated with low pressure, low temperature, and no salt byproduct.

In this paper, we report a solution synthesis of $\mathrm{Ge}^{0}$ nanowires from a newly characterized $\mathrm{Ge}^{+2}$ precursor, $\mathrm{Ge}(\mathrm{DBP})_{2}(\mathbf{1})$ where DBP represents $\mathrm{OC}_{6} \mathrm{H}_{3}\left(\mathrm{C}_{\left.\left(\mathrm{CH}_{3}\right)_{3}\right)_{2}-2,6 \text {. Our approach }}\right.$ hinges on molecularly designed precursors to control their reactivity and resulting nanostructure morphology. This conceptual approach has been introduced in the synthesis of several other systems such as $\mathrm{CdSe},{ }^{33} \mathrm{PbSe}$, and Au 34,35 Previously, we described the utility of $\mathrm{Ge}\left(\mathrm{N}\left(\mathrm{SiMe}_{3}\right)_{2}\right)_{2}$ to produce $\mathrm{Ge}^{0}$ nanocrystals. ${ }^{31}$ Herein, we tailor the precursor reactivity by the use of $\mathbf{1}$ to form $\mathrm{Ge}^{0}$ nanowires. The synthesis and characterization of both $\mathbf{1}$ and the subsequent $\mathrm{Ge}^{0}$ nanowires are described below.

\section{Experimental Details}

The synthesis was conducted at 285 to $315^{\circ} \mathrm{C}$ under $1 \mathrm{~atm}$ of $\mathrm{Ar}$ in a vacuum/Schlenk line setup, typically used for solution-synthesis of nanoparticles and nanowires. ${ }^{36}$ Note: no metal 
catalysts were used, and no salt byproducts were formed. All anhydrous solvents were purchased in sure-seal bottles. The following chemicals were purchased from Aldrich and used without further purification: $\mathrm{GeCl}_{2} \bullet$ dioxane, $\mathrm{LiN}\left(\mathrm{SiMe}_{3}\right)_{2}$, and oleylamine. $\mathrm{Ge}\left(\mathrm{N}\left(\mathrm{SiMe}_{3}\right)_{2}\right)_{2}$ was synthesized following literature reports. ${ }^{37}$

Fourier transform infrared (FTIR) transmission spectra were obtained on a Bruker Vector 22 spectrometer using $\mathrm{KBr}$ pellets pressed under an Ar atmosphere and handled under an atmosphere of flowing $\mathrm{N}_{2}$. Elemental analyses were performed on a Perkin-Elmer $2400 \mathrm{CHN}$ S/O elemental analyzer. Thermogravimetry analysis and differential thermal analysis (TGA/ DTA) were performed on a TA Instrument STD 2960 at $15{ }^{\circ} \mathrm{C} / \mathrm{min}$ ramp rate under Ar atmosphere. Nuclear magnetic resonance (NMR) data were collected on flame sealed samples dissolved in toluene- $d_{8}$ using a Bruker $250 \mathrm{MHz}$ NMR.

\section{$\mathrm{Ge}\left(\mathrm{OC}_{6} \mathrm{H}_{4}\left(\mathrm{C}\left(\mathrm{CH}_{3}\right)_{3}\right)-2,6\right)_{2}(1)$}

A solution of DBP-H $(0.525 \mathrm{~g}, 2.54 \mathrm{mmol})$ dissolved in toluene was slowly added by pipette to a stirring solution of $\mathrm{Ge}\left(\mathrm{N}\left(\mathrm{SiMe}_{3}\right)_{2}\right)_{2}(0.500 \mathrm{~g}, 1.27 \mathrm{mmol})$ in toluene. The mixture was heated to $50^{\circ} \mathrm{C}$ for $15 \mathrm{~min}$ to drive the reaction into completion and then allowed to cool to room temperature. X-ray quality pale yellow crystals of $\mathbf{1}$ were isolated by slow evaporation of the volatile component of the mixture. Yield: $0.335 \mathrm{~g}(67.0 \%)$. NMR: ${ }^{1} \mathrm{H}(400.1 \mathrm{MHz}$, tol$\left.d_{8}\right) \delta$ 7.37-7.29 (br m, 3-H, $\left.\mathrm{OC}_{6} \mathrm{H}_{3}\left(\mathrm{C}\left(\mathrm{CH}_{3}\right)_{3}\right)_{2}\right)$ and $\left.\left.1.54\left(\mathrm{~s}, 18 \mathrm{H}, \mathrm{OC}_{6} \mathrm{H}_{3}\left(\mathrm{C}_{(\mathrm{CH}}\right)_{3}\right)_{2}\right)\right) .{ }^{13} \mathrm{C}$ $\left\{{ }^{1} \mathrm{H}\right\}\left(100.0 \mathrm{MHz}\right.$, tol- $\left.d_{8}\right) \delta 140.6,125.9\left(\right.$ partial, $\left.\mathrm{OC}_{6} \mathrm{H}_{3}\left(C\left(\mathrm{CH}_{3}\right)_{3}\right)_{2}\right), 35.44\left(\mathrm{OC}_{6} \mathrm{H}_{3}(\mathrm{C}\right.$ $\left.\left.\left(\mathrm{CH}_{3}\right)_{3}\right)_{2}\right)$ ), and $\left.32.9\left(\mathrm{OC}_{6} \mathrm{H}_{3}\left(\mathrm{C}\left(\mathrm{CH}_{3}\right)_{3}\right)_{2}\right)\right)$. FTIR $\left(\mathrm{KBr}, \mathrm{cm}^{-1}\right)$ : 3088(s), 2965(sh, s), $2380(\mathrm{~s})$, 2044(s), 1914(s), 1859(s), 1794(s), 1696(s), 1641(s), 1578(s), 1469(sh, s), 1414(m), 1359(sh, s), 1316(s), 1218(m), 1109(m), 1019(s), 844(m), 746(m), 659(s), and 583(s). Elemental analysis calculated for $\mathrm{C}_{20} \mathrm{H}_{26} \mathrm{GeO}_{2}: 69.59 \% \mathrm{C}$ and $8.76 \% \mathrm{H}$. Found $69.36 \% \mathrm{C}$ and $8.78 \% \mathrm{H}$.

General X-ray Crystal Structure Information-Using a Bruker AXS diffractometer, a single crystal of $\mathbf{1}$ was mounted onto a thin glass fiber from a pool of Fluorolube ${ }^{\mathrm{TM}}$ and immediately placed under a liquid $\mathrm{N}_{2}$ stream. The radiation source was graphitemonochromatized Mo K $\alpha(\lambda=0.7107 \AA)$. The lattice parameters were optimized from a leastsquares calculation on carefully centered reflections. Lattice determination and data collection were carried out using SMART Version 5.054 software. Data reduction was performed using SAINT Version 6.01 software. The structure refinement was performed using XSHELL 3.0 software. The data were corrected for absorption, using the SADABS program within the SAINT software package. General collection parameters for $\mathbf{1}$ are shown in Table 1. Additional information concerning the structure of these compounds can be found in the supporting information section or by accessing the final CIF files through the Cambridge Crystallographic Data Base.

The structure was solved using direct methods, which yielded the heavy atoms, along with a number of the $\mathrm{C}$ and $\mathrm{O}$ atoms. Subsequent Fourier synthesis yielded the remaining atom positions. The $\mathrm{H}$ atoms were fixed in positions of ideal geometry and refined within the XSHELL software. These idealized $\mathrm{H}$ atoms had their isotropic temperature factors fixed at 1.2 or 1.5 times the equivalent isotropic $\mathrm{U}$ of the $\mathrm{C}$ atoms to which they were bonded. The final refinement of each compound included anisotropic thermal parameters on all non-hydrogen atoms. Final crystal solutions that contain alkoxides are well known to possess ligand positional disorder issues. Additional information concerning the data collection and final structural solutions of $\mathbf{1}$ can be found in the supporting information section.

Ge $\mathbf{0}^{\mathbf{0}}$ nanowire synthesis: In a vial, $\mathbf{1}(0.393 \mathrm{~g}, 1.00 \mathrm{mmol})$ was dissolved in oleylamine (2.69 $\mathrm{g}, 10.0 \mathrm{mmol}$ ) and stirred until all the material was completely dissolved forming a pale yellow solution. In general, this precursor solution was rapidly injected into a 3-neck flask containing 
non-coordinating solvent 1 -octadecene $(11.5 \mathrm{~mL}, 17.0 \mathrm{mmol})$ heated to $300{ }^{\circ} \mathrm{C}$. The temperature was measured by a K-type thermocouple and regulated with a variable voltage regulator (Variac) heater by an Omega positive-feedback temperature controller $9100 \mathrm{~A}$ with $3{ }^{\circ} \mathrm{C}$ accuracy. Upon injection, the temperature decreased to $280{ }^{\circ} \mathrm{C}$ and recovered to $300{ }^{\circ} \mathrm{C}$ within $1 \mathrm{~min}$, wherein the color of the solution changed immediately to dark purple. At selected intervals, $1 \mathrm{~mL}$ aliquots were taken and immediately quenched in $\sim 5 \mathrm{~mL}$ of $\mathrm{CHCl}_{3}$. These samples were used for TEM analyses. After the reaction was completed in $60 \mathrm{~min}$, the solution was extracted twice with a 50:50 vol.\% mixture of $\mathrm{CHCl}_{3} / \mathrm{CH}_{3} \mathrm{OH}$, and the final $\mathrm{Ge}^{0}$ nanowires in $\mathrm{CHCl}_{3}$ were then precipitated by the addition of excess acetone. After centrifugation, the dark $\mathrm{Ge}^{0}$ nanowires in a powder form can be rediluted in a variety of solvents, including $\mathrm{CHCl}_{3}$, toluene, and hexanes.

Characterization-JEOL 2010 high-resolution transmission electron microscope (HRTEM) with acceleration voltage of $200 \mathrm{KeV}$ was used to image $\mathrm{Ge}^{0}$ nanowires and to obtain their selected area electron diffraction (SAED) pattern. TEM sample preparation consisted of dissolving $\mathrm{Ge}^{0}$ nanowires in $\mathrm{CHCl}_{3}$, toluene, or hexane. The dissolution was followed by dispersing a drop of the solution on to a holey-carbon copper grid and allowing it to dry completely. In addition, the powder was characterized by $\mathrm{Cu} \mathrm{K} \alpha$ radiation from a Siemens 500 diffractometer with 0.2-degree step size and 10-second dwell time. The $\mathrm{Ge}^{0}$ nanowire sample was placed on a zero background X-ray diffraction (XRD) holder, which minimized the background noise against XRD signal.

\section{Results and Discussion}

We have previously demonstrated the formation of $\mathrm{Ge}^{0}$ nanoparticles through the reduction of $\mathrm{Ge}^{+2}$ amide precursor $\mathrm{Ge}\left(\mathrm{N}\left(\mathrm{SiMe}_{3}\right)_{2}\right)_{2}{ }^{31}$ Our approach was to design $\mathrm{Ge}^{+2}$ precursors at the molecular level, thus controlling their reactivity and resulting morphologies. Following this approach, we sought alternative $\mathrm{Ge}^{+2}$ precursors that could be tailored to generate $\mathrm{Ge}^{0}$ nanowires instead of particles. A number of important characteristics, such as general structure, pendant ligand chains, decomposition temperature, and solubility, were considered in designing the precursors. For $\mathrm{Ge}^{0}$ nanoparticles, we reasoned that due to the high reactivity of $\mathrm{Ge}\left(\mathrm{N}\left(\mathrm{SiMe}_{3}\right)_{2}\right)_{2}$, numerous nucleation sites were formed upon injection. ${ }^{31}$ This led to rapid consummation of the precursor and caused uncontrolled growth of nanoparticles. ${ }^{38}$ In order to obtain a nanowire morphology, it is critical to control the rate of nucleation site generation, while maintaining sufficient precursor feedstock to continue the 1-D growth. ${ }^{38}$ Two main factors that affect the reactivity of the precursor are the Ge-ligand bond strength and the steric hindrance of the ligand. Therefore, we chose alkoxides as the precursor, whose Ge-O dissociation energy is approximately $157 \mathrm{kcal} / \mathrm{mol} .{ }^{39}$ This dissociation energy is much greater than $55 \mathrm{kcal} / \mathrm{mol}^{39}$ of the $\mathrm{Ge}-\mathrm{N}$ bond in $\mathrm{Ge}\left(\mathrm{N}\left(\mathrm{SiMe}_{3}\right)_{2}\right)_{2}$. Pendant chain manipulation is also much easier with alkoxides than with amides due to the larger number of alcohols readily available.

\section{Precursor Synthesis}

Numerous ligands, such as $\mathrm{OR}=\mathrm{OC}\left(\mathrm{C}\left(\mathrm{CH}_{3}\right)_{3}\right)_{2},{ }^{40} \mathrm{OC}_{6} \mathrm{H}_{2}\left(\mathrm{C}\left(\mathrm{CH}_{3}\right)_{3}\right)_{2}-2,6\left(\mathrm{CH}_{3}\right)-4,{ }^{41}$ $\mathrm{OC}_{6} \mathrm{H}_{3}\left(\mathrm{CH}_{3}\right)_{3}-2,4,6,{ }^{42} \mathrm{OC}_{6} \mathrm{H}_{3}\left(\mathrm{CH}\left(\mathrm{CH}_{3}\right)_{2}\right)_{2}-2,6,{ }^{42} \mathrm{OC}_{6} \mathrm{H}_{3}\left(\mathrm{C}_{6} \mathrm{H}_{5}\right)_{2}-2,6,{ }^{42}$ and $\mathrm{OC}_{6} \mathrm{H}_{3}\left(\mathrm{C}_{6} \mathrm{H}_{5}\right)_{4}-2,3,5,642$ were found to be useful for isolation of solvent-free $\mathrm{Ge}(\mathrm{OR})_{2}$. However, the absence of a systematic variation in the ligands made it difficult to correlate ligand effects on the final material physical properties. Therefore, we undertook the systematic synthesis of $\mathrm{Ge}(\mathrm{OR})_{2}$ to tailor the reactivity, using a variety of alkoxide ligands produced from an amide alcohol metathesis route shown in eq 1 . The majority of this family of $\mathrm{Ge}(\mathrm{OR})_{2}$ compounds will be reported shortly; however, the dibutylphenoxide (DBP) derivative proved 
to have the characteristics that were favored for nanowire growth and will be discussed exclusively at this time.

$$
\mathrm{Ge}\left(\mathrm{NR}_{2}\right)_{2}+2 \mathrm{H}-\mathrm{DBP} \rightarrow \mathrm{Ge}(\mathrm{DBP})_{2}+2 \mathrm{HNR}_{2}
$$

Compound $\mathbf{1}$ was synthesized according to eq 1 and the structure plot of $\mathbf{1}$ is shown in Figure 1. Upon addition of the alcohol to the pale yellow solution of $\mathrm{Ge}\left(\mathrm{NR}_{2}\right)_{2}$ in toluene, the reaction mixture turned darker yellow. After stirring for several minutes, the reaction was heated to ensure that complete exchange had occurred, while no additional changes in color were noted. The reaction was then allowed to cool to room temperature and set aside until X-ray quality crystals were formed.

The FTIR transmission spectrum of the bulk powder indicated that the amide was fully substituted by the alkoxides. The structure of $\mathbf{1}$ was solved by single crystal X-ray analysis as a mononuclear species with a bent geometry and is similar to the literature derivatives. ${ }^{41}$ The bond distances and angles were found to be in agreement with the literature structures. $40-42$ Elemental analysis confirmed that the bulk sample was consistent with single crystal structure. Since the thermogravimetry analysis (TGA) data of $\mathbf{1}$ (supplement 1 ) revealed a decomposition temperature around $200{ }^{\circ} \mathrm{C}$, it was necessary to use solvents with a boiling point higher than this temperature for nanomaterial production. The noncoordinating 1-octadecene was selected as the solvent. 31

\section{$\mathrm{Ge}^{0}$ Nanowires}

The TEM image of typical $\mathrm{Ge}^{0}$ nanowires isolated after synthesis is shown in Figure 2a. The SAED pattern shown in inset of Figure 2a displays typical diffraction spots of $\{111\}$ and $\{220\}$ planes, which indicate that the $\mathrm{Ge}^{0}$ nanowires are in cubic phase. The nanowires' anisotropic growth can reach up to $10 \mu \mathrm{m}$ in length, while the diameter ranges from 15 to $25 \mathrm{~nm}$.

Observations around the nanowires also reveal the presence of smaller nanorods, which can be attributed to the arrested growth of the nanorods that formed during the initial nucleation stage. The HRTEM images in Figure $2 \mathrm{~b}$ and $2 \mathrm{c}$ indicate that the nanowires are crystalline. The HRTEM image in Figure $2 b$ shows lattice fringes with d-spacing of $3.26 \AA$, which corresponds to $\{111\}$ planes, growing in [110] direction. A corresponding diffraction pattern obtained by fast Fourier transformation (FFT) is shown in the inset. Similarly, another HRTEM image in Figure $2 \mathrm{c}$ also shows visible lattice fringes of the $\{111\}$ plane but with [111] growth direction. These directions are consistent with the reported growth direction of $\mathrm{Ge}^{0}$ nanowires via other methods. Two XRD measurements, conducted immediately after the synthesis (Figure 3), show the presence of $\{111\},\{220\},\{311\},\{400\}$, and $\{331\}$ planes of the cubic phase $\mathrm{Ge}^{0}$ nanowires. The presence of these XRD peaks after the 60-day exposure to the ambient air suggests that the $\mathrm{Ge}^{0}$ nanowires are well passivated by the amine surfactant, which minimizes surface oxidation of $\mathrm{Ge}^{0}$ nanowires. The adsorption of amines on the surface of $\mathrm{Ge}$ has been previously demonstrated. 31,43 In comparison to the FTIR transmission spectrum of oleylamine (Figure 4a), the FTIR transmission spectrum of the $\mathrm{Ge}^{0}$ nanowires, taken after washing with $\mathrm{CHCl}_{3}$ and $\mathrm{CH}_{3} \mathrm{OH}$, reveals the presence of characteristic peaks of amine surfactant, 31,43 such as $\mathrm{N}-\mathrm{H}$ stretching at $3460 \mathrm{~cm}^{-1}$ and N-H scissor modes at $1640 \mathrm{~cm}^{-1}$ (Figure $4 \mathrm{~b}$ ). This result further verifies that the $\mathrm{Ge}^{0}$ nanowires are encapsulated by oleylamine on the surface.

The dimensions of $\mathrm{Ge}^{0}$ nanowires were found to depend strongly on both the time and temperature of the reaction. Figure 5 captures the temporal evolution of $\mathrm{Ge}^{0}$ nanowires over the temperature range from 285 to $315^{\circ} \mathrm{C}$. When the synthesis was conducted at a low temperature $\left(285^{\circ} \mathrm{C}\right)$, no definitive shape formation was observed (Figure 5a) after $5 \mathrm{~min}$; however, after $60 \mathrm{~min}, \mathrm{Ge}^{0}$ rods $\leq 50 \mathrm{~nm}$ in length with $6 \mathrm{~nm}$ in diameter were observed (Figure $5 b)$. At a higher temperature $\left(300^{\circ} \mathrm{C}\right), \mathrm{Ge}^{0}$ rods, whose diameter and length are $10-17 \mathrm{~nm}$ 
and $20-50 \mathrm{~nm}$, respectively, formed within $5 \mathrm{~min}$ (Figure 5c). After $60 \mathrm{~min}$, the length of $\mathrm{Ge}^{0}$ nanowires was an order of magnitude longer $(200-400 \mathrm{~nm})$, while maintaining the 20$\mathrm{nm}$ diameter (Figure 5d). Increasing the temperature even higher to $315^{\circ} \mathrm{C}$ resulted in the formation of substantially longer $\mathrm{Ge}^{0}$ nanowires with the 20 -nm diameter. The length of these nanowires increased from $400 \mathrm{~nm}$ ( $5 \mathrm{~min}$, Figure $5 \mathrm{e}$ ) to $10 \mu \mathrm{m}$ (60 min, Figure 5f), depending on the reaction time. As the temperature is increased, the axial growth of the nanowires is favored over the radial growth. The formation of the $\mathrm{Ge}^{0}$ nanowires in this system is influenced not only by the reactivity of $\mathbf{1}$, but also by the surfactant passivation. The role of surfactant in solution growth of nanowires has been investigated in other systems, ${ }^{44}$ wherein amine surfactant acts as a reducing agent and a molecular capping agent. The lone electron pair in the amines provides the necessary electron charge for the reduction process, which can be observed by the change from yellowish to dark color during the reaction. We speculate that upon mixing of oleylamine with $\mathbf{1}$, an intermediate Ge compound is formed, containing both Ge-N and Ge-O bonds. The color of the compound after mixing remains constant, which suggests that there is no change in the oxidation state of the Ge precursor. Upon injection into the boiling solvent, several reactions most likely occur simultaneously, including the reduction of $\mathbf{1}$ by the amine, dissociation of Ge-N and Ge-O bonds, and nucleation of $\mathrm{Ge}^{0}$ nanostructure. The higher bond strength of Ge-O bonds and the steric hindrance from the ligands increase the activation energy barrier for the reduction process, which translates to higher temperature needed to reduce the precursor and to start the nucleation process. The precursor reactivity governs the production rate of nucleation sites. To support this mechanism, a control experiment was conducted by replacing the oleylamine surfactant with 1-dodecanethiol surfactant. The result showed that no reduction process occurred because of the high bond strength of Ge-S and suggested that amine surfactant is a more suitable surfactant. ${ }^{45,46}$ The complete reduction of $\mathbf{1}$ into $\mathrm{Ge}^{0}$ was confirmed by the repeatable observation of lattice fringes in the TEM images (Figure 2) and XRD diffraction peaks (Figure 3) that are consistent with $\mathrm{Ge}^{0}$ and not with $\mathrm{GeO}_{\mathrm{x}}$. The excess amine surfactant present in the system served as a capping agent around the nanowires. Mui et al. have shown that methylamine chemisorbs on the $\mathrm{Ge}$ (100) surface in the gas phase without undergoing N-H dissociation. ${ }^{43}$ Instead of dissociative adsorption, the lone pair electrons of the amines bond to the $\mathrm{Ge}^{0}$ surface.

\section{Nanowires growth mechanism}

Understanding the growth mechanism of these $\mathrm{Ge}^{0}$ nanowires is necessary in order to garner greater control over the nanowire dimensions. From temporal investigations, we observed two potential growth mechanisms: (a) self-seeding (Figure 6a) mechanism and (b) self-assembly mechanism (Figure 6b). Both mechanisms showed approximately equal probability of occurrence.

For the self-seeding mechanism, we examined the diffraction patterns generated by (FFT of selected areas in the TEM images of the wire [inset of Figure 6a (i-iii)] to determine whether the head (seed) and tail (wire) exist as a single crystal. The FFT images revealed that the head (i), the interface (ii), and the tail (iii) share a common crystallographic plane (111), which indicate that they are epitaxially connected, single-crystalline $\mathrm{Ge}^{0}$. The contrast between the head and the tail observed in the TEM images is due to the difference in their thickness.

The image in Figure 7a is a snapshot of the self-seeding crystal formation taken after 5 min, where two crystalline $\mathrm{Ge}^{0}$ nanorods are overlapping. One rod functions as a seed with arrested growth, while the other continues to grow as a wire. Extending the reaction time to $60 \mathrm{~min}$, the head and tail configuration clearly emerges with the wire growing in [100] direction (Figure $7 b$ ). These observations support that $\mathrm{Ge}^{0}$ nanowires grow in low Miller index directions, such as [100], [110], and [111]. ${ }^{47}$ The diameter of the seed as well as the wire is maintained, indicating that the surface of the seed is well passivated by the surfactant. The HRTEM image 
of the seed taken at a tilt angle (inset of Figure 7b) reveals that the seed is single-crystalline $\mathrm{Ge}^{0}$. From these images, the self-seeding growth mechanism appears to be similar to the VLS growth mechanism, except that the $\mathrm{Ge}^{0}$ serves as its own catalyst (self-seeded).

In contrast, a self-assembly growth mechanism (Figure 6b) was also noted, where an aggregate of smaller rods can be seen near the tip, but not along the side of the nanowires. The commonly observed presence of these aggregates suggests that they recrystallize on the tips to extend the length of $\mathrm{Ge}^{0}$ nanowires. The TEM image in Figure $8 \mathrm{a}$ is another snapshot taken during the reaction, where several $\mathrm{Ge}^{0}$ nanorods aggregate and cause the nanowire to grow anisotropically. From the image, we observe a clear outline of individual single-crystalline nanorods that over time, these nanorods align themselves in the growth direction, start to recrystallize, and form domains of single-crystalline nanowires. The HRTEM image of a section along the wire is shown in Figure 8b. The lattice fringes in Figure $8 \mathrm{~b}$ as well as FFT images in the inset (i-iii) indicate that the recrystallization occurs at the boundaries between nanorods and an individual wire, forming a single-crystalline $\{111\}$ domain along the $\mathrm{Ge}^{0}$ nanowires. From this result, we propose that the Ge monomers in solution insert themselves at the interface of coalescing nanorods and/or at the interface between a rod and a wire. Similar nanowire growth mechanisms have been observed in the self-assembly of ellipsoid $\mathrm{Ag}^{0}$ nanocrystals to form $\mathrm{Ag}^{0}$ nanowires. ${ }^{48}$ Figure 9 offers further support of the self-assembly mechanism. In inset (i), a $\mathrm{Ge}^{0}$ nanowire longer than $5 \mu \mathrm{m}$ has been formed with nanorod aggregates still present at the tip. The SAED image in inset (ii) taken from the tip of the nanowire shows the presence of multi-domain, single-crystalline nanorods with $\{111\},\{220\}$, and $\{311\}$ planes. The SAED image in inset (iii) taken from the section along the nanowire represents a later stage in the wire growth, where these nanorods recrystallize into a growing single-crystalline $\mathrm{Ge}^{0}$ nanowire.

\section{Conclusions}

The simple solution synthesis of $\mathrm{Ge}^{0}$ nanowires via the use of $\mathrm{Ge}^{+2}$ precursor has been described. The main reason for using molecularly designed $\mathrm{Ge}^{+2}$ precursors is that their reduction potential is significantly lower than that of $\mathrm{Ge}^{+4} .49$ We found that with less energy required to reduce the precursor to $\mathrm{Ge}^{0}$, the synthesis can be conducted at lower temperatures $\left(300-315{ }^{\circ} \mathrm{C}\right)$ and pressure $(1 \mathrm{~atm})$ under Ar without the use of any potentially contaminating seed materials.

Precursor $\mathbf{1}$ is found to play a crucial role in the formation of $\mathrm{Ge}^{0}$ nanowires in this system. The stronger Ge-ligand bond and increased steric hindrance assist in decreasing the precursor reactivity, which limits the degree of nucleation and allows for controlled nanowire growth. We surmise that the free lone-pair electrons on the nitrogen facilitates both the reduction of $\mathrm{Ge}^{+2}$ as well as the surface passivation of nanowires by the amine. Higher temperatures accelerate the growth and result in longer nanowires.

Two growth mechanisms are observed in this system: (i) self-seeding mechanism and (ii) selfassembly mechanism. Self-seeding mechanism relies on the overlap between two $\mathrm{Ge}^{0}$ moieties at the initial stage of growth, providing a growth platform for subsequent nanowire growth. The lower potential of the overlapping area serves as a sink for the monomer to be incorporated into the growing nanowire. In the self-assembly mechanism, the nanowire growth occurs when the nanorods aggregate at the tip of the nanowire and undergo recrystallization process to form a single-crystalline material, while being incorporated into the growing plane of the $\mathrm{Ge}^{0}$ nanowires. 


\section{Supplementary Material}

Refer to Web version on PubMed Central for supplementary material.

\section{Acknowledgement}

The authors would like to acknowledge Ying Bing Jiang for his help with the TEM images and NSF CAREER (DMR-0094145) for the partial support of this project. This work was partially funded by the National Institutes of Health (NIH) through the NIH Roadmap for Medical Research, Grant \#1 R21 EB005365-01. Information on this RFA (Innovation in Molecular Imaging Probes) can be found at

http://grants.nih.gov/grants/guide/rfa-files/RFA-RM-04-021.html. Sandia is a multiprogram laboratory operated by Sandia Corporation, a Lockheed Martin Company, for the United States Department of Energy under contract DEAC04-94AL85000.

\section{References}

1. Li LS, Alivisatos AP. Adv. Mater 2003;15:408-411.

2. Cui Y, Lieber CM. Science 2001;291:851-853. [PubMed: 11157160]

3. Cui Y, Wei QQ, Park HK, Lieber CM. Science 2001;293:1289-1292. [PubMed: 11509722]

4. Alivisatos AP. Science 1996;271:933-937.

5. Brus LE. J. Chem. Phys 1983;79:5566-5571.

6. Wu YY, Yang PD. Chem. Mat 2000;12:605-607.

7. Wang DW, Dai HJ. Angew. Chem. Int. Ed 2002;41:4783-4786.

8. Heath J, Legoues F. Chem. Phys. Lett 1993;208:263-268.

9. Omi H, Ogino T. Appl. Phys. Lett 1997;71:2163-2165.

10. Zhang YF, Tang YH, Wang N, Lee CS, Bello I, Lee ST. Phys. Rev. B 2000;61:4518-4521.

11. Sze, SM. Physics of Semiconductor Devices. 2nd ed.. New York: John Wiley \& Sons, Inc.; 1981.

12. Maeda Y, Tsukamoto N, Yazawa Y, Kanemitsu Y, Masumoto Y. Appl. Phys. Lett 1991;59:31683170.

13. Bostedt C, van Buuren T, Willey TM, Franco N, Terminello LJ, Heske C, Moller T. Appl. Phys. Lett 2004;84:4056-4058.

14. Melnikov DV, Chelikowsky JR. Solid State Commun 2003;127:361-365.

15. Takagahara T, Takeda K. Phys. Rev. B 1992;46:15578-15581.

16. Rossetti R, Hull R, Gibson JM, Brus LE. J. Chem. Phys 1985;83:1406-1410.

17. Gerion D, Zaitseva N, Saw C, Casula MF, Fakra S, Van Buuren T, Galli G. Nano Lett 2004;4:597602.

18. Mathur S, Shen H, Sivakov V, Werner U. Chem. Mat 2004;16:2449-2456.

19. Morales AM, Lieber CM. Science 1998;279:208-211. [PubMed: 9422689]

20. Hanrath T, Korgel BA. J. Am. Chem. Soc 2002;124:1424-1429. [PubMed: 11841311]

21. Ryan KM, Erts D, Olin H, Morris MA, Holmes JD. J. Am. Chem. Soc 2003;125:6284-6288. [PubMed: 12785861]

22. Ziegler KJ, Polyakov B, Kulkarni JS, Crowley TA, Ryan KM, Morris MA, Erts D, Holmes JD. J. Mater. Chem 2004;14:585-589.

23. Audoit G, Ni Mhuircheartaigh T, Lipson SM, Morris MA, Blau WJ, Holmes JD. J. Mater. Chem 2005;15:4809-4815.

24. Crowley TA, Daly B, Morris MA, Erts D, Kazakova O, Boland JJ, Wu B, Holmes JD. J. Mater. Chem 2005; 15:2408-2413.

25. Hanrath T, Korgel BA. Adv. Mater 2003;15:437-440.

26. Shah PS, Hanrath T, Johnston KP, Korgel BA. J. Phys. Chem. B 2004;108:9574-9587.

27. Hanrath T, Korgel BA. J. Am. Chem. Soc 2004;126:15466-15472. [PubMed: 15563174]

28. Wagner RS, Ellis WC. Appl. Phys. Lett 1964;4:89-90.

29. Zaitseva N, Harper J, Gerion D, Saw C. Appl. Phys. Lett 2005;86:053105. 
30. Lu XM, Fanfair DD, Johnston KP, Korgel BA. J. Am. Chem. Soc 2005;127:15718-15719. [PubMed: 16277510]

31. Gerufng H, Bunge SD, Boyle TJ, Brinker CJ, Han SM. Chem. Commun 2005:1914-1916.

32. Xia YN, Yang PD, Sun YG, Wu YY, Mayers B, Gates B, Yin YD, Kim F, Yan YQ. Adv. Mater 2003;15:353-389.

33. Peng ZA, Peng XG. J. Am. Chem. Soc 2001;123:183-184. [PubMed: 11273619]

34. Green M, O'Brien P. Chem. Commun 2000:183-184.

35. Hampden-Smith, MJ.; Kodas, TT. The Chemistry of Metal CVD. 1st ed.. New York: VCH; 1994.

36. Murray CB, Norris DJ, Bawendi MG. J. Am. Chem. Soc 1993;115:8706-8715.

37. Davidson, Peter J.; Harris, David H.; Lappert, MF. J. Am. Chem. Soc. - Dalton Trans 1976:22682274.

38. Yu WW, Wang YA, Peng XG. Chem. Mat 2003;15:4300-4308.

39. Lappert, MF.; Power, PP.; Sanger, AR.; Srivastava, RC. Metal and Metalloid Amides. 1st ed.. Chichester: Halsted Press; 1980.

40. Fjeldberg T, Hitchcock PB, Lappert MF, Smith SJ, Thorne AJ. J. Chem. Soc.-Chem. Commun 1985:939-941.

41. Cetinkaya B, Gumrukcu I, Lappert MF, Atwood JL, Rogers RD, Zaworotko MJ. J. Am. Chem. Soc 1980;102:2088-2089.

42. Weinert CS, Fenwick AE, Fanwick PE, Rothwell IP. Dalton Trans 2003:532-539.

43. Mui C, Han J, Wang G, Musgrave C, Bent S. J. Am. Chem. Soc 2002;124:4027-4038. [PubMed: 11942841]

44. Law M, Goldberger J, Yang PD. Ann. Rev. Mater. Res 2004;34:83-122.

45. Kosuri MR, Cone R, Li QM, Han SM, Bunker BC, Mayer TM. Langmuir 2004;20:835-840. [PubMed: 15773112]

46. Han SM, Ashurst WR, Carraro C, Maboudian R. J. Am. Chem. Soc 2001;123:2422-2425. [PubMed: 11456892]

47. Mullin, JW. Crystallization. 3rd ed.. Great Britain: Butterworth-Heinemann Ltd.; 1993.

48. Korgel BA, Fitzmaurice D. Adv. Mater 1998;10:661-665.

49. Bard, AJ.; Parsons, R.; Jordan, J. Standard Potentials in Aqueous Solutions. 1st ed. New York: IUPAC (Marcel Dekker); 1985. 


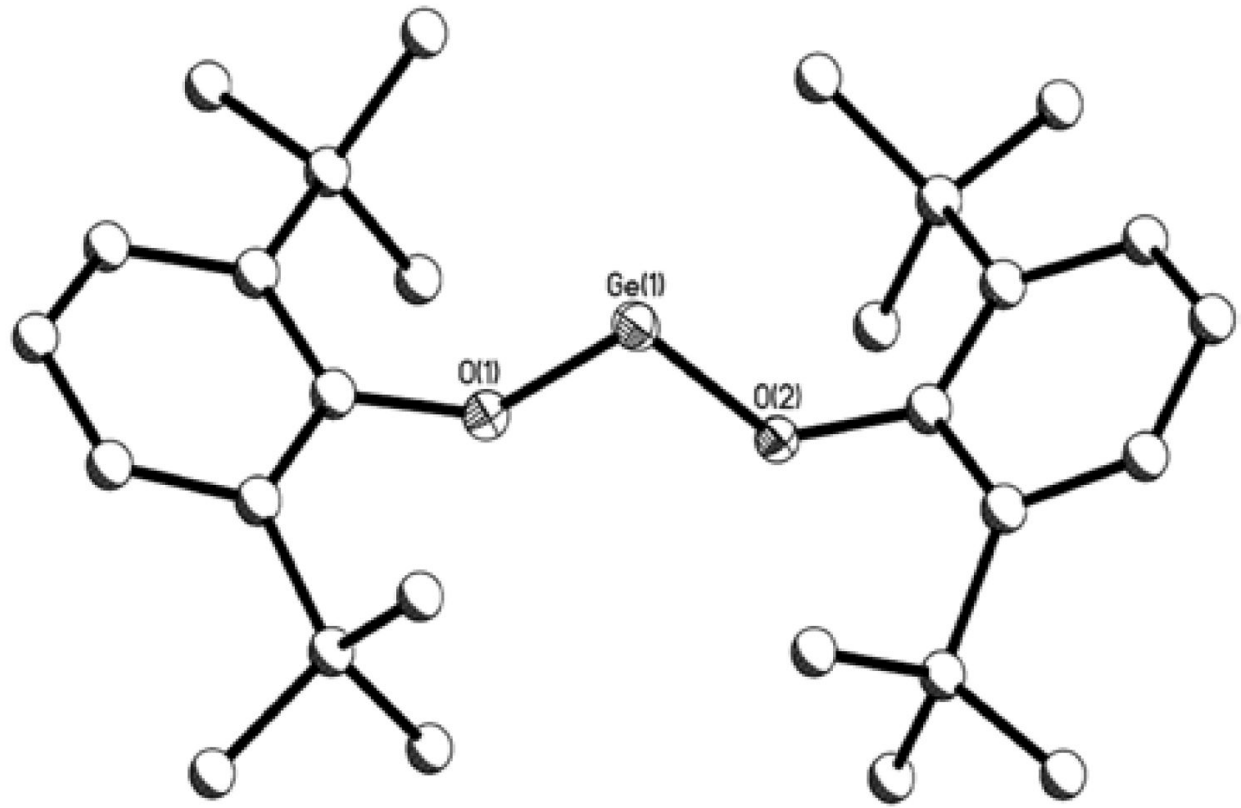

Figure 1.

Structure plot of $\mathbf{1}$. Thermal ellipsoids of heavy atoms plot is drawn at $30 \%$ level. Carbons are shown as ball and stick for clarity. 

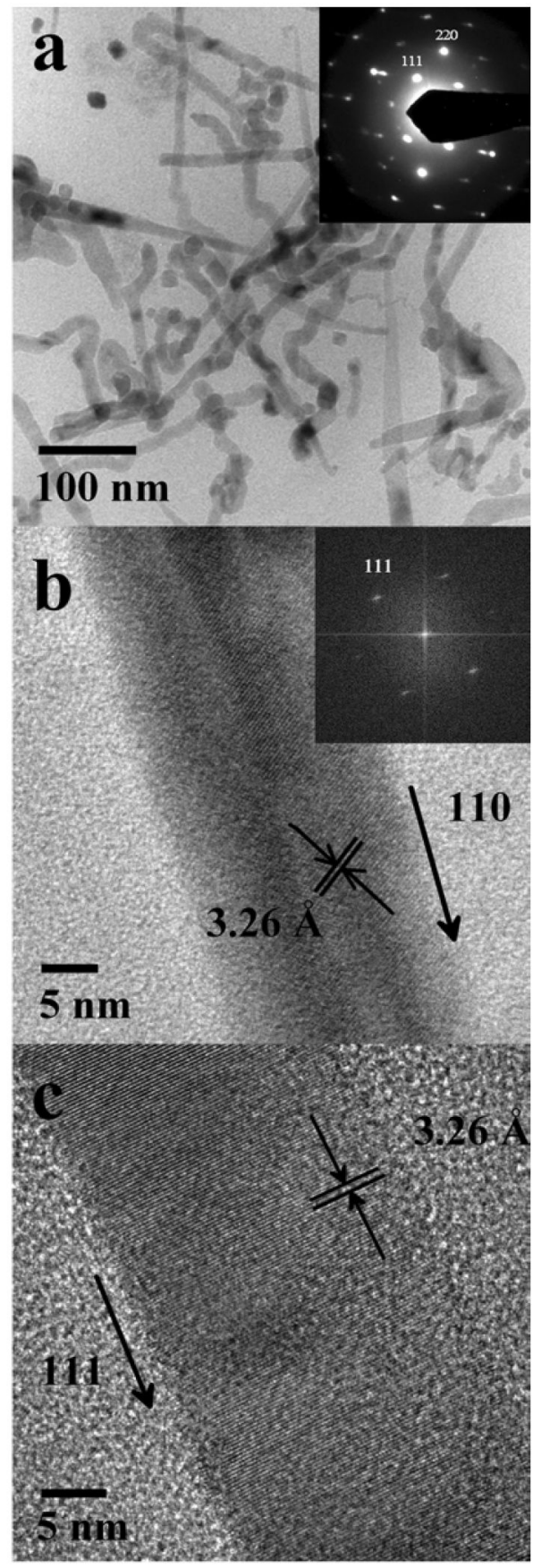

Figure 2.

(a) $\mathrm{Ge}^{0}$ nanowires (inset: typical SAED pattern), (b) HRTEM image of $\mathrm{Ge}^{0}$ nanowires in [110] direction (inset: FFT image), (c) HRTEM image of $\mathrm{Ge}^{0}$ nanowires in [111] direction. 


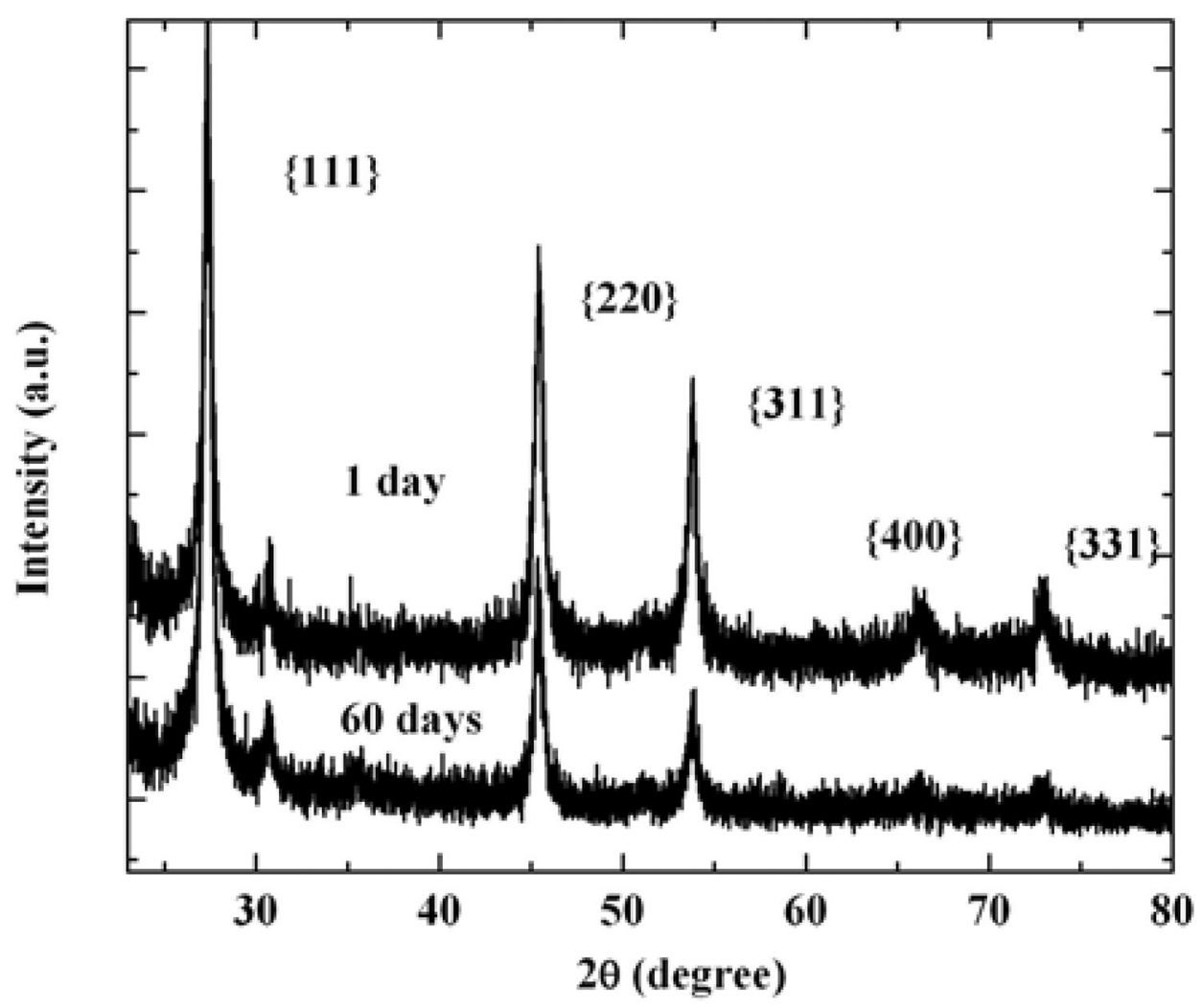

Figure 3.

XRD of $\mathrm{Ge}^{0}$ nanowires shows the cubic structure and the stability in air after 60 days. 


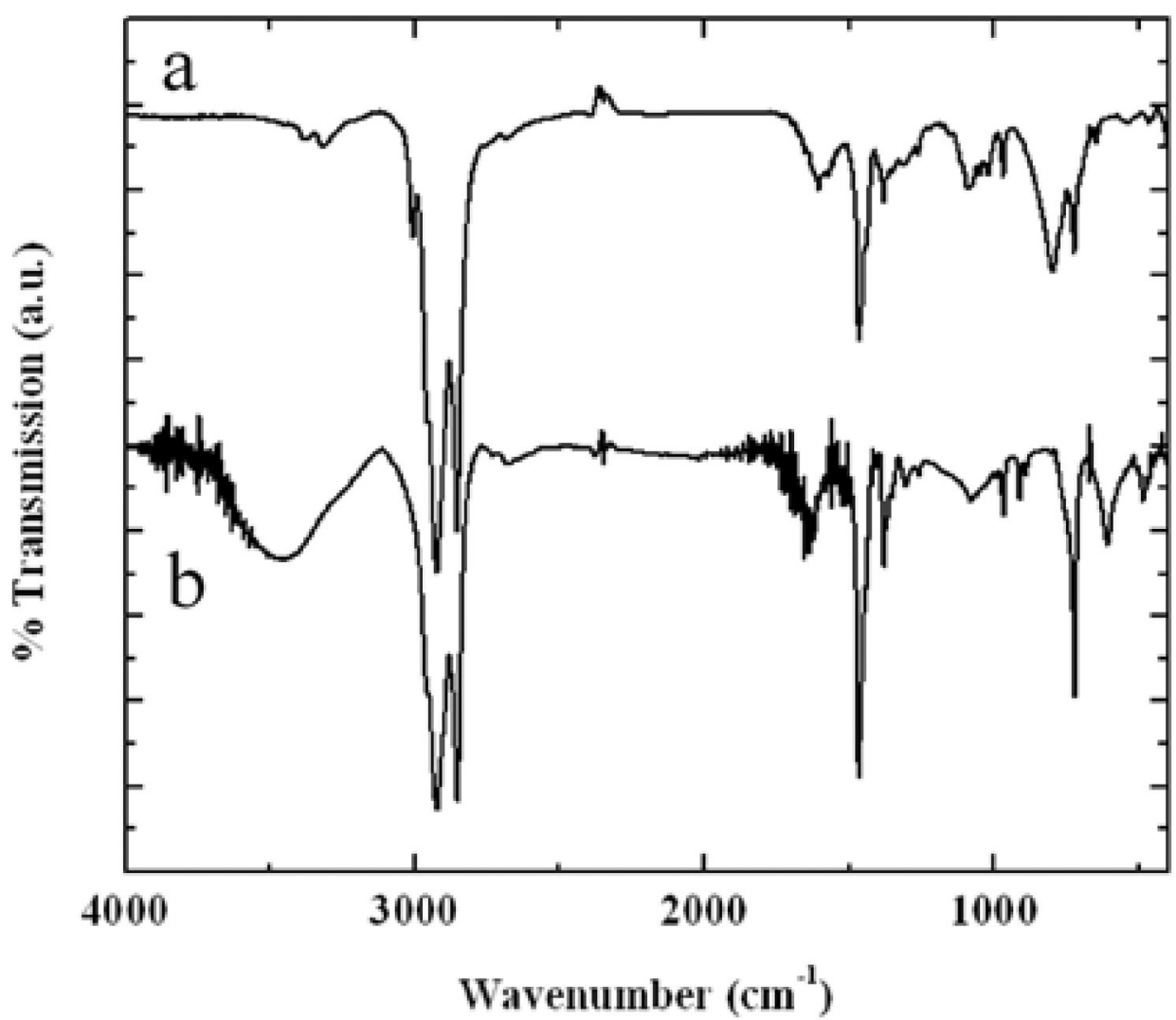

Figure 4.

(a) FTIR transmission spectrum of oleylamine, (b) FTIR transmission spectrum of $\mathrm{Ge}^{0}$ nanowires. 

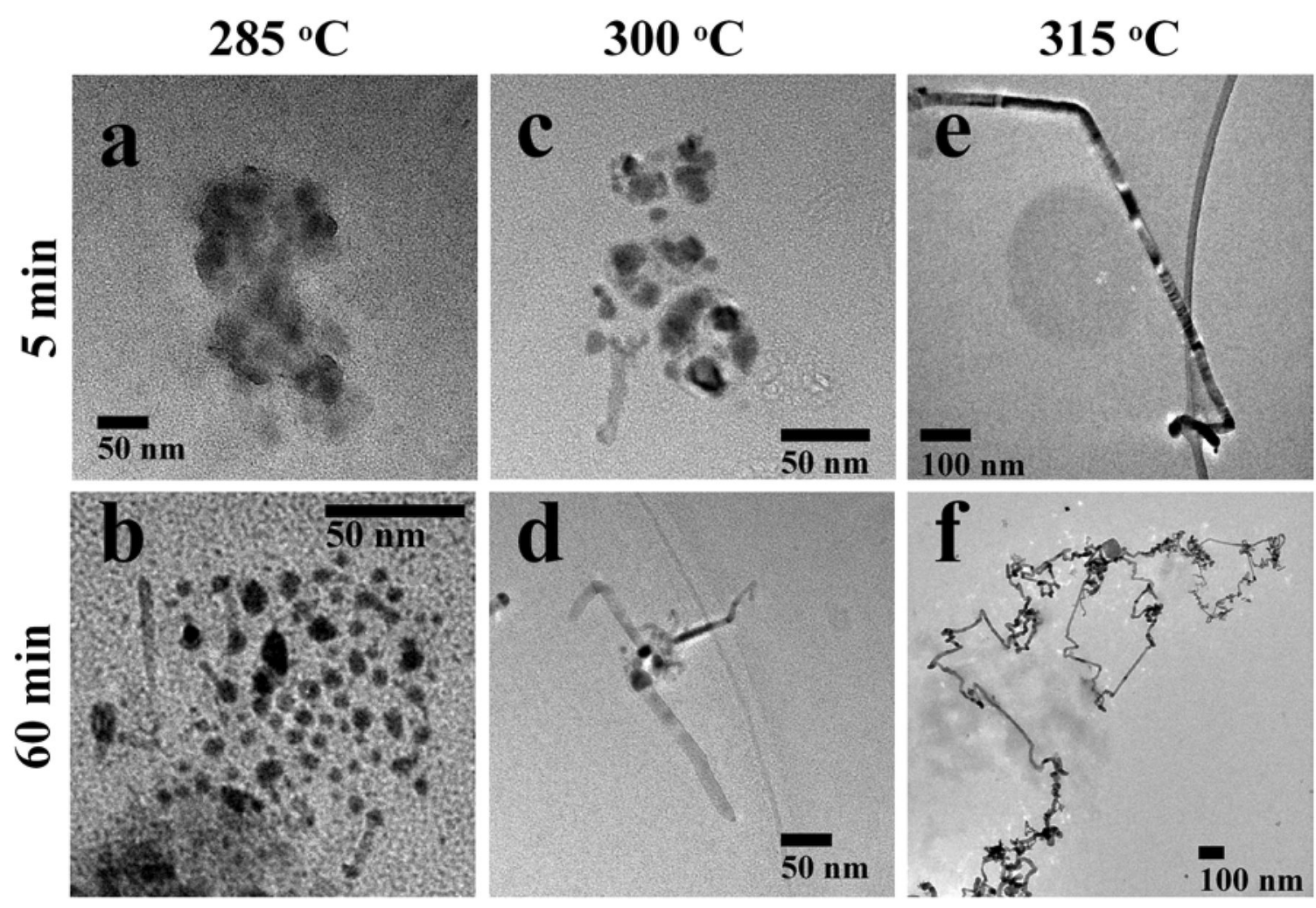

Figure 5.

The effect of temperature on the growth of $\mathrm{Ge}^{0}$ nanowires. At $285^{\circ} \mathrm{C}$ after (a) 5 min and (b) $60 \mathrm{~min}$, at $300{ }^{\circ} \mathrm{C}$ after (c) $5 \mathrm{~min}$ and (d) $60 \mathrm{~min}$, and at $315^{\circ} \mathrm{C}$ after (e) $5 \mathrm{~min}$ and (f) $60 \mathrm{~min}$. 

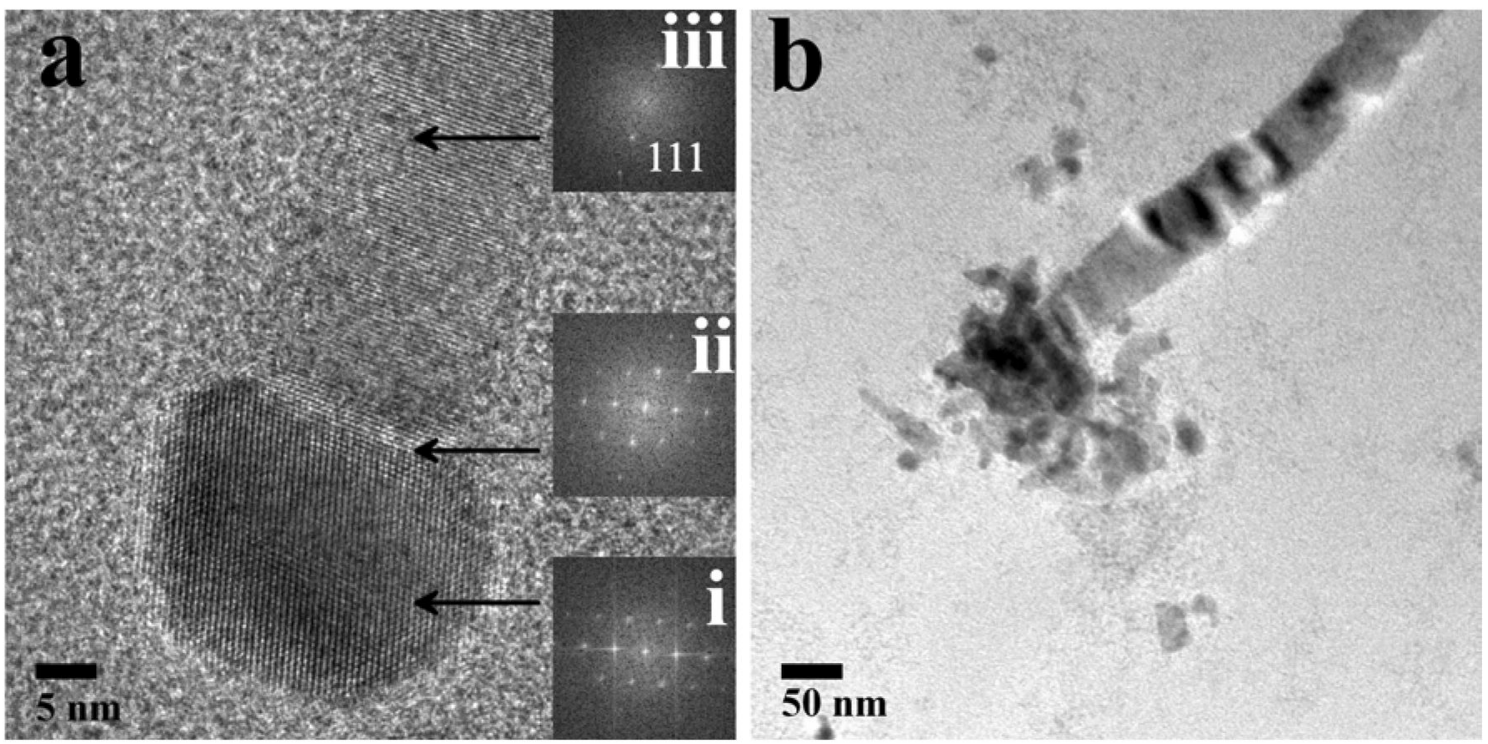

Figure 6.

Two different growth mechanisms are observed: (a) self-seeding mechanism with the diffraction patterns of the head (inset (i)), the interface (inset (ii)) and the tail (inset (iii)) shows $\{111\}$ planes, and (b) self-assembly growth showing aggregates of nanorods at the tip of the nanowire. 

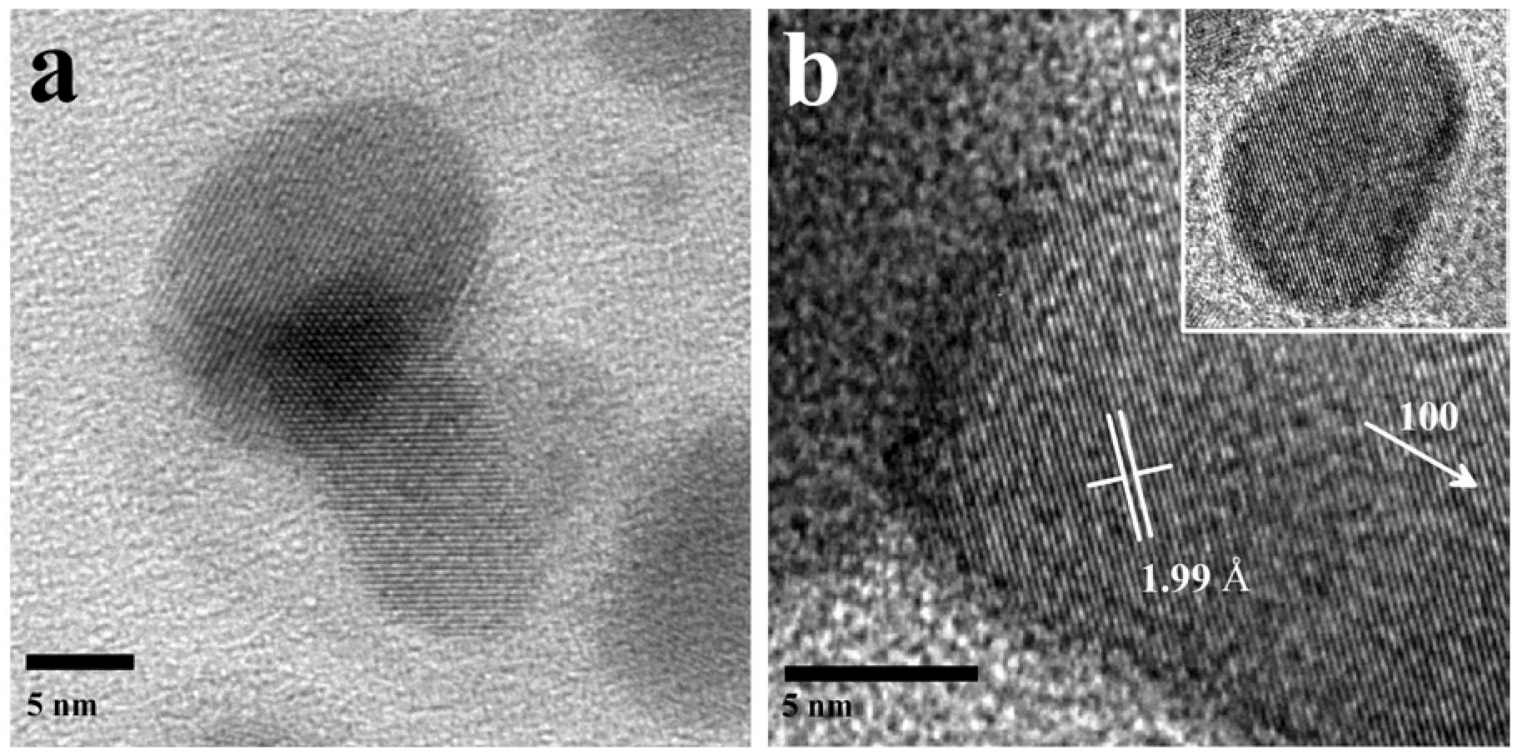

Figure 7.

(a) The snapshot shows an overlap between two $\mathrm{Ge}^{0}$ nanorods. (b) One of the rods continues to grow in [100] direction to form a nanowire. 


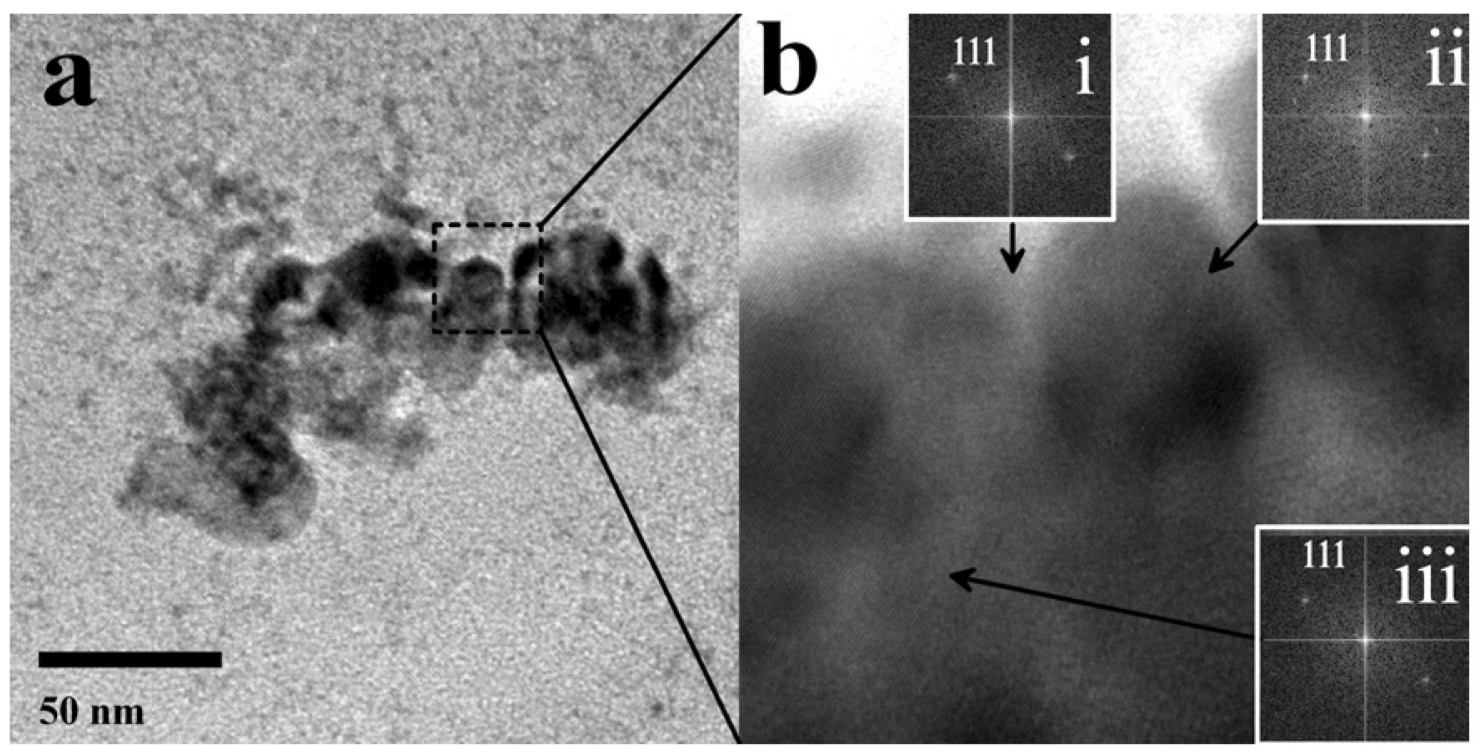

Figure 8.

(a) Self-assembly process of an aggregate of $\mathrm{Ge}^{0}$ nanorods. (b) HRTEM image indicating formation of $\{111\}$ planes across the nanorods. Inset (i), (ii), and (iii) show the diffraction patterns of $\{111\}$ planes across the nanorods. 


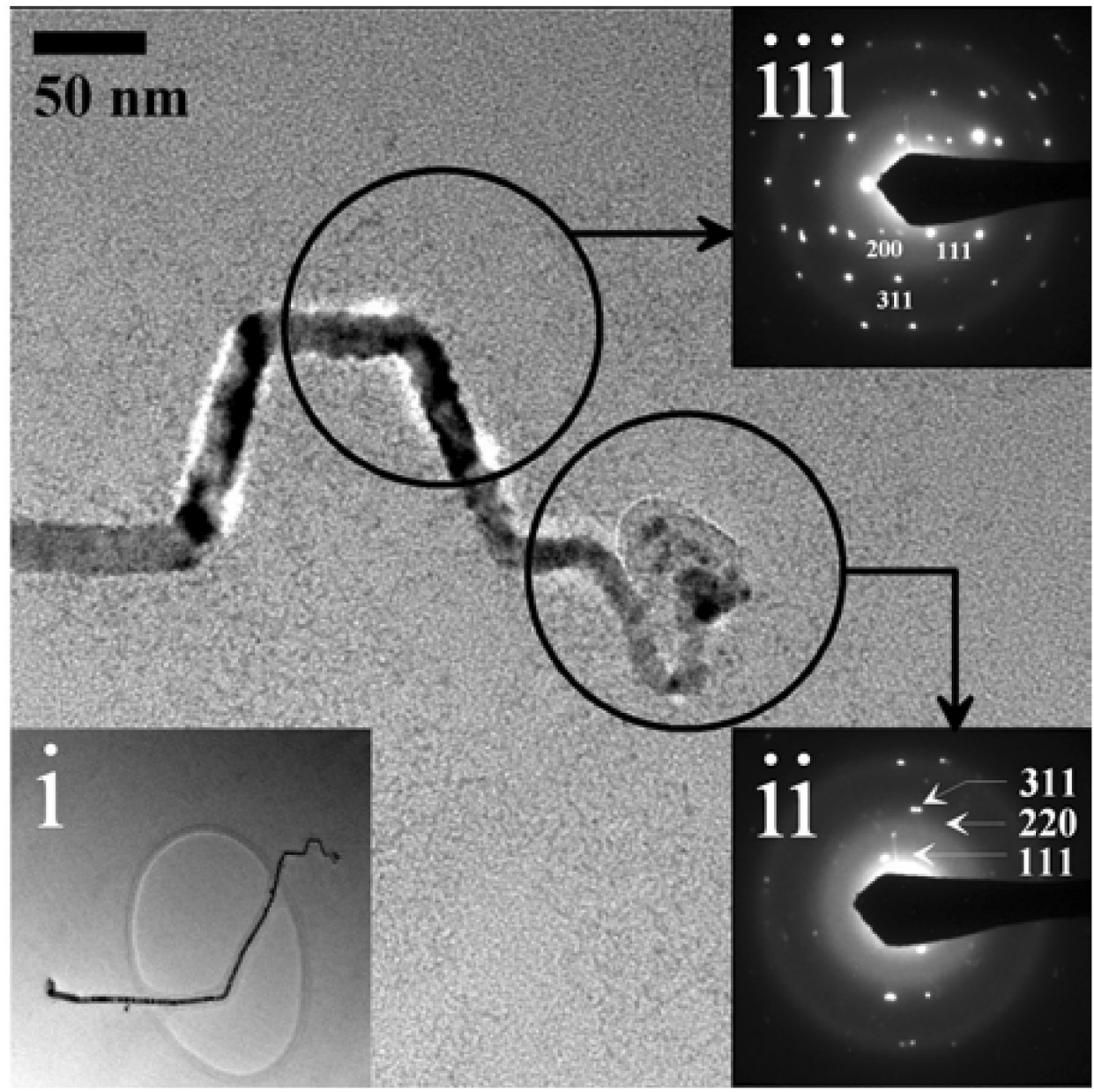

Figure 9.

Self-assembly at the nanowire tip: (i) low resolution TEM image, (ii) SAED of the tip, and (iii) SAED of the nanowire. 
Data collection parameters for $\mathbf{1}$

Table 1

\begin{tabular}{ll}
\hline formula & $\mathrm{C}_{28} \mathrm{H}_{42} \mathrm{Ge}_{2}$ \\
formula weight & 483.21 \\
crystal system & Monoclinic \\
space group & $\mathrm{P} 2(1) / \mathrm{n}$ \\
$a, \AA$ & $13.620(4)$ \\
$b, \AA$ & $13.810(4)$ \\
$c, \AA$ & $14.056(4)$ \\
$\beta$, deg & $95.534(5)$ \\
$\mathrm{V}, \AA^{3}$ & $2631.3(12)$ \\
$\mathrm{Z}$ & 4 \\
$\mathrm{~T}(\mathrm{~K})$ & $203(2)$ \\
calculated density, $\mathrm{mg} / \mathrm{m}^{3}$ & 1.220 \\
absorption coefficient, $\mathrm{mm}^{-1}$ & 1.185 \\
number of reflections & 18605 \\
number of observed reflections & 4774 \\
$\mathrm{R} 1^{a}$ (\%) (all data) & $6.16(9.54)$ \\
wR2 $^{b}$ (\%) (all data) & $13.92(15.86)$
\end{tabular}

$a_{R_{1}}=\Sigma\left\|\mathrm{F}_{0}|-| F_{C}\right\| \Sigma\left|F_{0}\right| \times 100$.

$\left.b_{w R 2}=\mid \Sigma w\left(F_{0}^{2}-F_{c}^{2}\right)^{2} / \Sigma\left(w\left|F_{0}\right|^{2}\right)^{2}\right]^{1 / 2} \times 100$ 